'Fundação Oswaldo Cruz (Fiocruz), Escola Nacional de Saúde Pública Sergio Arouca (Ensp), Laboratório de Avaliação de Situaç̃es Endêmicas Regionais (Laser) - Rio de Janeiro (RJ), Brasil.

beatriz.soares28@gmail.com

2 Fundação Oswaldo Cruz (Fiocruz), Escola Nacional de Saúde Pública Sergio

Arouca (Ensp), Laboratório de Avaliação de Situações Endêmicas Regionais (Laser) - Rio de Janeiro (RJ), Brasil. giselacardoso@ensp.

fiocruz.br

${ }^{3}$ Fundação Oswaldo Cruz (Fiocruz), Escola Nacional de Saúde Pública Sergio Arouca (Ensp), Laboratório de Avaliação de Situações Endêmicas Regionais (Laser) - Rio de Janeiro (RJ), Brasil.

anaclaudiafigueiro@ensp.

fiocruz.br

\section{Análise estratégica da Vigilância Epidemiológica em tuberculose: uma experiência local}

\author{
Strategic analysis of tuberculosis Epidemiological Surveillance: a \\ local experience
}

Beatriz da Costa Soares ${ }^{\mathbf{1}}$, Gisela Cordeiro Pereira Cardoso², Ana Cláudia Figueiró $\mathbf{3}$

RESUMO Esta análise estratégica teve por objetivo apreciar a adequação das ações de Vigilância Epidemiológica de tuberculose em município prioritário do estado do Rio de Janeiro. É um estudo de caso que emprega métodos mistos envolvendo revisão bibliográfica, análise documental, observação direta e entrevista semiestruturada com profissionais de saúde e gestores. As evidências indicam que, apesar de as ações serem realizadas conforme as recomendações nacionais, não têm sido suficientes para o controle da doença localmente em razão da adoção de perspectiva restrita de Vigilância Epidemiológica e da dificuldade dos profissionais de saúde de incorporar tais ações.

PALAVRAS-CHAVE Tuberculose. Avaliação em saúde. Vigilância Epidemiológica.

ABSTRACT This strategic analysis aimed at assessing the adequacy of Tuberculosis Epidemiological Surveillance actions in a priority municipality in the State of Rio de Janeiro. The case study employed mixed methods involving literature review, document analysis, direct observation and semi-structured interview with health professionals and health managers. Evidences indicate that, in spite of the actions have been carried out following national recommendations, they are not sufficient for the local control of the disease. This may be due to both the adoption of a limited perspective of Epidemiological Surveillance activities and the difficulty of health professionals to incorporate such actions.

KEYWORDS Tuberculosis. Health evaluation. Epidemiological Surveillance. 


\section{Introdução}

A tuberculose (TB) é, ainda hoje, entendida como grave problema de saúde pública, e o Programa de Controle da Tuberculose (PCT) tem nas ações de Vigilância Epidemiológica (VE) um braço estratégico de prevenção e controle da doença (BRASIL, 2011). A análise estratégica configura-se como importante ferramenta para apreciar a suficiência e adequação da intervenção em relação aos problemas apresentados pelo contexto local. Estudos desse tipo são escassos na literatura, embora auxiliem os gestores na tomada de decisão. Isso significa dizer que tais estudos têm a função de avaliar se a intervenção utilizada foi a mais apropriada para o contexto em questão e, em caso negativo, permitir o direcionamento para o que seria mais adequado para a situação (BROUSSELLE ET AL., 2011).

\section{Contexto epidemiológico da TB}

No Relatório Global sobre a TB, a Organização Mundial da Saúde (OMS, 2014) relaciona o Brasil como integrante do grupo constituído pelos 22 países com a maior carga da doença, concentrando $82 \%$ dos casos mundiais. O Brasil ocupava, no mesmo ano, a $16^{\mathrm{a}}$ posição no grupo em relação ao número de casos novos de TB.

No contexto nacional, estudos revelam que o estado do Rio de Janeiro era o segundo mais afetado em relação ao número de casos da doença até o ano de 2013. Nesse mesmo ano, o município de Itaboraí, onde se desenvolveu este estudo, apresentava altos coeficientes de incidência da doença (69 casos novos/100.000 habitantes), ultrapassando as taxas apresentadas pelo estado do Rio de Janeiro (61,7 casos novos/100.000 habitantes). O estado, por sua vez, possui $32 \mathrm{mu}-$ nicípios prioritários para o controle da TB, dentre eles o de Itaboraí (BRASIL, 2015).

No município de Itaboraí, a coordenação das ações e serviços prestados aos indivíduos acometidos pela doença segue as recomendações nacionais por meio da implementação do PCT. O programa de Itaboraí foi premiado algumas vezes como modelo de combate à doença. Dentre os prêmios, está o da Organização Pan-Americana da Saúde (Opas) de 2005 por apresentar dados de cura e abandono dentro das metas estabelecidas pela OMS, que são de $5 \%$ para abandono e $85 \%$ para cura (BRASIL, 2011). As taxas, porém, não se mantiveram nos últimos anos.

As diretrizes do PCT recomendam a descentralização das medidas de controle para a Atenção Básica (AB), aumentando o acesso da população em geral e dos grupos vulneráveis ao acometimento pela doença, tais como população de rua, pessoas privadas de liberdade, população indígena, dentre outras. $\mathrm{O}$ PCT incentiva, ainda, o fortalecimento do controle social, a sustentabilidade das ações de controle e a promoção da equidade na atenção aos pacientes (BRASIL, 2011).

$\mathrm{O}$ documento nacional regulamentador das ações relacionadas à TB - Manual de recomendações do Ministério da Saúde para o controle da TB (2011) - orienta a ação visando à interrupção da cadeia de transmissão da doença e à redução da possibilidade de adoecimento por meio da busca de casos, do diagnóstico precoce e adequado e do tratamento até a cura. Para tal, o programa é dividido em componentes que devem facilitar o planejamento, a execução e a avaliação das ações de controle da doença.

Dentre esses componentes, encontra-se a 'informação estratégica', que abrange a VE da TB. Através dela, é possível conhecer a magnitude da doença a partir dos dados de morbimortalidade, sua distribuição, fatores de risco e tendência no tempo e espaço, dando subsídios para as ações de controle e auxiliando na prevenção da doença (BRASIL, 2011; SOARES, 2015). De acordo com o mesmo manual, as principais ações de vigilância para o controle da TB são: a) a estruturação da busca ativa e confirmação de casos; b) a comunicação imediata dos casos por meio da notificação ao Sistema de Informação 
de Agravos de Notificação (Sinan) (BRASIL, 2015); c) a investigação dos contatos e a investigação diagnóstica para confirmação de casos; d) o acompanhamento do tratamento e o encerramento dos casos; e) a notificação nos outros possíveis sistemas de informação em saúde; f) o auxílio à assistência realizado conjuntamente às visitas domiciliares a casos novos e a convocação de faltosos; e g) o monitoramento dos exames laboratoriais.

Contudo, a vigilância, tal como proposta por Castellanos (1991), deve compreender a investigação causal da doença, a procura por explicações da situação de saúde em grupos específicos da população, avaliando os contextos e a relação mais ou menos complexa destes com a situação de saúde dos grupos populacionais. Isso significa dizer que a vigilância deve ser a responsável por compreender que cada grupo específico possui uma lógica diferenciada de produção e reprodução das doenças, utilizando-se da investigação e análise do contexto local para descobrir a origem da disseminação ou intensidade das doenças e agravos (CASTELLANOS, 1991).

Dessa forma, o estudo buscou compreender se a VE em TB de Itaboraí segue estritamente às recomendações nacionais para o controle da doença ou se segue uma perspectiva ampliada de vigilância, como a apresentada por Castellanos (1991), contemplando as análises de contexto, os indicadores de vulnerabilidade e a compreensão e intervenção nos problemas detectados.

\section{Contexto social da TB}

A doença ainda é associada, prioritariamente, às baixas condições de vida e renda. Problemas tais como crescimento populacional, população moradora de rua, dependência química, precárias condições de moradia, má alimentação, baixa renda, falta de saneamento básico, dentre outras questões, estão diretamente associadas à incidência da doença. Em um município como Itaboraí, marcado pelas precárias condições de vida e saúde da população, esse perfil de pessoas acometidas pela doença se acentuou (SIQUEIRA, 2014).

Itaboraí possui uma população de aproximadamente 218.000 habitantes, dos quais $37 \%$ sobrevivem com até $1 / 2$ salário mínimo e apenas $21 \%$ vive em domicílios com saneamento adequado. Além dessas questões, Itaboraí recebeu em 2006 o início da construção do Complexo Petroquímico do Rio de Janeiro (Comperj), o que provocou intensa transformação do espaço urbano local e impactou os serviços de saúde (SIQUEIRA, 2014).

Dado o cenário, a VE pode constituir-se num espaço de definição de problemas e prioridades do território, bem como de atuação sobre a realidade local, por meio das análises de situação de saúde de maneira conjunta com os diferentes setores de governo e da população (OLIVEIRA; CASANOVA, 2009). A vigilância deve ser o espaço de percepção da rede de causalidades dos problemas de saúde para a intervenção no território com ações integrais e intersetoriais.

\section{Análise estratégica}

A avaliação em saúde tem por objetivos fundamentais auxiliar no planejamento e elaboração de intervenções ou no fornecimento de informações para a melhoria das mesmas, determinando seus efeitos e decidindo sobre sua manutenção ou transformação. Ao incentivar a transformação de uma situação injusta ou problemática, a avaliação contribui para o progresso do conhecimento e para a elaboração teórica (ALVES ET AL., 2010).

A análise estratégica pode ser uma etapa da avaliação ou a própria avaliação em si, que se atém à análise de pertinência da intervenção. A pertinência de uma intervenção depende, fundamentalmente, do contexto - seja ele epidemiológico, sociocultural, sanitário, financeiro, político ou geográfico - dos atores envolvidos e do alinhamento entre objetivos e interesses, nos quais estão mergulhadas as intervenções em análise (BROUSSELLE ET AL., 2011). 
Pode-se dizer que esse tipo de análise tem por função responder a uma série de questionamentos relacionados ao contexto, tais como se: a) a intervenção é coerente com a resolução do problema; b) a intervenção é a melhor ou a mais adequada diante do contexto existente; c) é pertinente intervir sobre o problema, considerando sua importância e o impacto da intervenção; d) é pertinente intervir de tal forma; e) a intervenção atende aos principais fatores determinantes do problema e à sua população-alvo; f) os objetivos definidos inicialmente são adequados para resolver o problema e responder às necessidades locais; e g) os responsáveis pela intervenção estão agindo adequadamente, considerando suas funções e atribuições (CARDOSO ET AL., 2016).

Estes fatores contextuais, por sua vez, estão relacionados a três principais perspectivas de análise. Em primeiro lugar, pode-se analisar a pertinência da escolha do problema, ou seja, se o problema sobre o qual atua a intervenção é, de fato, seu foco. Em segundo lugar está a análise sobre a escolha dos objetivos da intervenção. Nesse momento determina-se se o objetivo, ou causa, selecionado foi o mais pertinente dentre o conjunto de possibilidades existentes. Cabe aqui ressaltar que é nesse momento, também, que deve ser analisada a pertinência da escolha da população-alvo da intervenção. Em terceiro lugar, analisa-se, então, a pertinência das parcerias estratégicas. Isto é, se as parcerias selecionadas para a elaboração e execução de uma intervenção foram as mais adequadas para o cenário político, estratégico e institucional existente (BROUSSELLE ET AL., 2011; SOARES, 2015).

Por fim, dado que são procedimentos semelhantes e que se confundem, vale ressaltar a diferença entre o planejamento estratégico e a análise estratégica. O planejamento estratégico constitui-se num conjunto de técnicas e operações para atuar sobre determinado problema. No sentido contrário, a análise estratégica avalia se o planejamento realizado e a maneira como se executaram as ações foram as melhores escolhas para a situação. O planejamento é, portanto, realizado antes da intervenção e a análise estratégica, depois (BROUSSELLE ET AL., 2011).

Neste estudo, a análise estratégica empreendeu um julgamento quanto à adequação, conformidade ou harmonização das ações e práticas de VE realizadas pelo PCT de Itaboraí em relação à situação de saúde local. Isto é, verificou-se se havia sentido e correspondência entre as ações de saúde e a realidade local. Dessa forma, as perguntas avaliativas norteadoras para este estudo objetivaram (1) Conhecer as ações de VE da TB executadas em Itaboraí e se condizem com as recomendações nacionais; e (2) Avaliar se as ações realizadas pela VE são adequadas e suficientes em relação às necessidades apresentadas pela realidade local.

\section{Metodologia}

Trata-se de estudo de caso com emprego de métodos mistos, combinando metodologia qualitativa e quantitativa (GREENE; BENJAMIN; GOODYEAR, 2001) de maneira a triangular e complementar as informações acerca do objeto de estudo. As técnicas de coleta compreenderam revisão de literatura, análise documental, observação direta e entrevista semiestruturada, como pode ser verificado no quadro 1. O estudo foi realizado no biênio 2014-2015. 
Quadro 1. Técnicas e fontes para a coleta de informações

\begin{tabular}{|c|c|c|}
\hline Procedimento utilizado & Informação desejada & Fontes \\
\hline Revisão de Literatura & $\begin{array}{l}\text { Contexto local de Itaboraí, caracterização } \\
\text { do PCT e da TB. }\end{array}$ & $\begin{array}{l}\text { Bases de dados (Scientific Electronic Library } \\
\text { Online - SciELO, PubMed, periódicos Ca- } \\
\text { pes - Coordenação de Aperfeiçoamento de } \\
\text { Pessoal de Nível Superior), livros, Instituto } \\
\text { Brasileiro de Geografia e Estatística (IBGE), } \\
\text { Sinan }\end{array}$ \\
\hline Entrevistas & $\begin{array}{l}\text { Informações sobre o contexto local, a ca- } \\
\text { racterização do PCT e da TB, além de infor- } \\
\text { mações sobre as ações, funções, vantagens } \\
\text { e limitações da atuação da vigilância em TB. }\end{array}$ & $\begin{array}{l}\text { Relatos dos informantes-chave a partir de } \\
\text { roteiro semiestruturado }\end{array}$ \\
\hline Análise Documental & $\begin{array}{l}\text { Acompanhamento dos casos de TB; análise } \\
\text { da priorização da TB e da vigilância pela } \\
\text { SMS, seus objetivos, metas e indicadores; } \\
\text { análise da configuração, funções e ações do } \\
\text { PCT e da vigilância epidemiológica. }\end{array}$ & $\begin{array}{l}\text { Manual de Recomendações para o Controle } \\
\text { da TB; Livro de Acompanhamento de Ca- } \\
\text { sos; Relatório Anual de Gestão e Plano de } \\
\text { Enfrentamento de TB/HIV }\end{array}$ \\
\hline Observação Direta & $\begin{array}{l}\text { Reconhecimento e percepção da realidade } \\
\text { local e dos serviços de saúde para atenção } \\
\text { à TB. }\end{array}$ & $\begin{array}{l}\text { Observação e descrição do campo em } \\
\text { diário de campo }\end{array}$ \\
\hline
\end{tabular}

Em relação à análise documental, foram construídos roteiros para o Livro de Acompanhamento de Casos ('Livro Verde'), o Relatório Anual de Gestão (RAG) e o Plano de Enfrentamento de TB/HIV (vírus da imunodeficiência humana).

Para a realização das entrevistas, foram selecionados os informantes-chave para as categorias de estudo, quais sejam, profissionais da gestão, da referência secundária em TB e da AB em saúde. Esses atores foram selecionados por considerar-se que poderiam fornecer informações pertinentes relacionadas às ações de TB no município. Em relação aos profissionais de saúde da $\mathrm{AB}$, foram selecionados três enfermeiros de diferentes unidades da Estratégia Saúde da Família (ESF). O roteiro semiestruturado utilizado para direcionar tais entrevistas compreendia questões sobre as ações assistenciais e de vigilância em TB, sobre a organização, estrutura e proposta do PCT no município, além de questionar o perfil epidemiológico da doença e o contexto local.
A análise das entrevistas se deu através da transcrição e ordenamento do material em um quadro de perguntas e respostas por ator entrevistado. Elaborou-se uma tabela de categorias de convergência-divergência, agrupando as respostas de acordo com sua semelhança para formar categorias temáticas de análise (BARDIN, 2011).

A observação direta, por sua vez, foi realizada ao longo do estudo, durante as visitas aos serviços e unidades de saúde, e registrada em diário de campo sem roteiro estabelecido. A observação direta é um método de coleta de dados que consiste na utilização dos sentidos do observador, ou seja, na atuação de um observador para obter determinados tipos de informações, como comportamentos, eventos ou condições ambientais relevantes (POPE; MAYS, 2009).

Considerado suficiente o material coletado, foi elaborada uma matriz de informações. Nela, são indicadas as perguntas dos instrumentos que responderiam a cada uma das perguntas avaliativas do estudo. Como limitação deste trabalho, deve ser mencionada 
a inviabilidade temporal para entrevistar aos Agentes Comunitários de Saúde (ACS) e o subsecretário de VE.

O estudo foi aprovado pelo Comitê de Ética em Pesquisa da Escola Nacional de Saúde Pública Sergio Arouca (Ensp/ Fiocruz), sob o número de parecer 742.403 , de 29/07/2014. Não houve conflito de interesse neste estudo.

\section{Resultados e discussão}

Os achados deste estudo foram sistematizados visando responder às perguntas avaliativas da pesquisa. A figura 1 representa o modelo teórico-lógico da VE em TB. Essa figura apoiou as discussões, análises e conclusões relacionadas à pergunta sobre conhecer as ações de VE da TB executadas em
Itaboraí e se elas condizem com as recomendações nacionais.

O modelo teórico-lógico da figura 1 sintetiza e esquematiza as ações de VE preconizadas pelo PCT nacional, seus efeitos no curto, médio e longo prazo, relacionando-os com os fatores de contexto existentes no município de Itaboraí, ou seja, as condições socioeconômicas locais, as populações mais vulneráveis, assim como o cenário político e de implementação do polo petroquímico do Comperj. O modelo lógico permitiu a comparação entre o que é preconizado e o que é realizado no município para que seja possível, então, compreender se as ações realizadas estão de acordo com as recomendações nacionais. Ele fundamentou, ainda, a discussão sobre a adequação dessas ações frente ao contexto local.

Figura 1. Modelo teórico da Vigilância Epidemiológica em TB do município de Itaboraí (RJ), Brasil

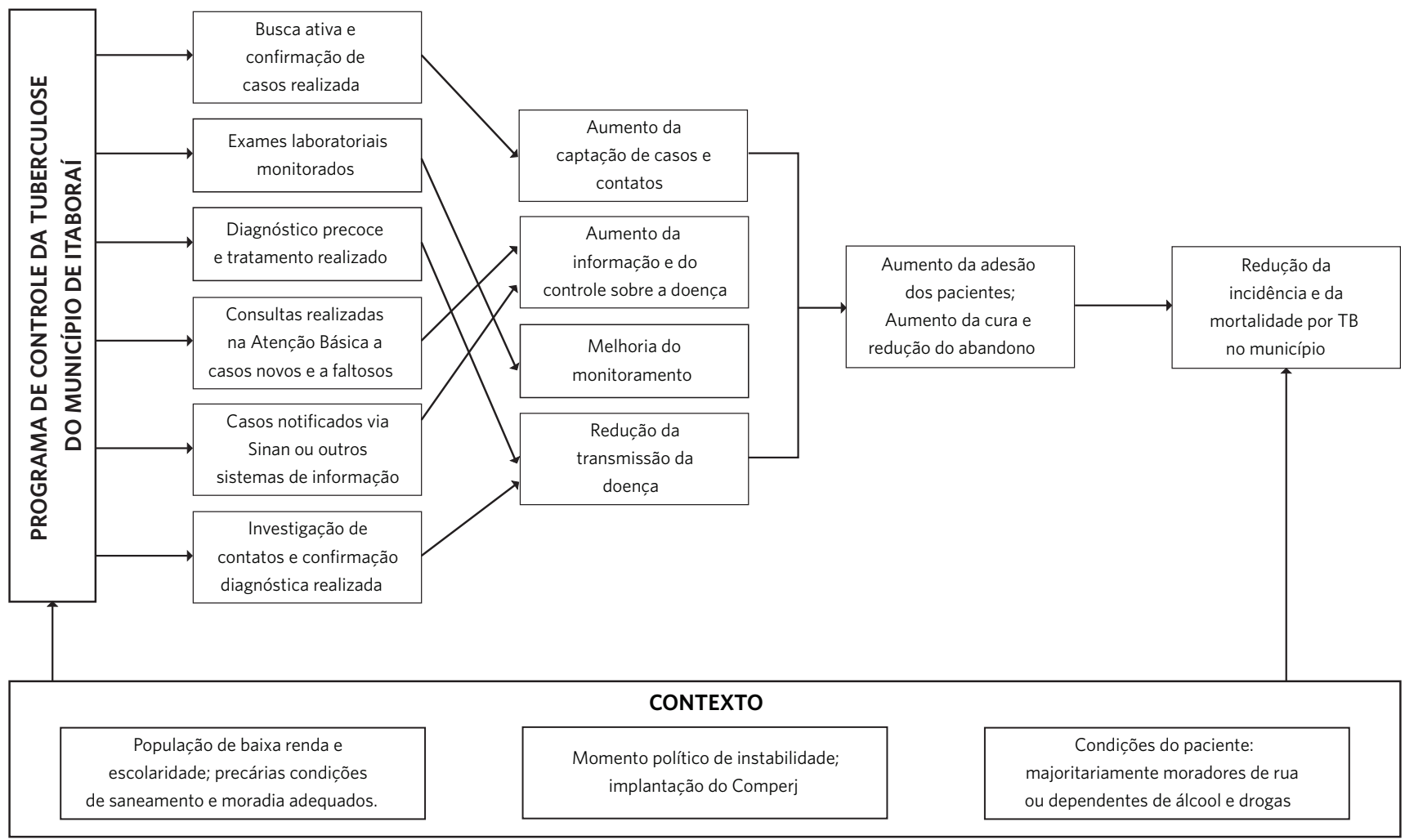


O quadro 2 apresenta a síntese das respostas às entrevistas por nível de atuação do entrevistado e critérios analisados. Apoia a resposta para ambas as perguntas avaliativas, embora sistematize principalmente as respostas à pergunta como conhecer se as ações realizadas pela VE são adequadas e suficientes em relação às necessidades apresentadas pela realidade local. O quadro tem por função apresentar as respostas dos informantes aos principais critérios em análise no estudo, identificando categorias, ou seja, grupos de semelhança entre as respostas, que possibilitem a compreensão do contexto e das ações de vigilância da TB no local segundo a perspectiva de cada um dos entrevistados. As respostas foram agrupadas a partir de dois eixos explorados pelo roteiro de entrevista: os fatores de contexto e o PCT local. Desse modo, é possível visualizar como as respostas estão impregnadas do papel e função dos atores entrevistados.

Quadro 2. Síntese das respostas aos instrumentos de entrevista por nível de atuação do entrevistado

\begin{tabular}{|c|c|c|c|c|}
\hline Eixos & Critérios & Gestão & Coordenação & $\begin{array}{l}\text { Unidade Saúde da Família } \\
\text { (USF) }\end{array}$ \\
\hline \multirow{6}{*}{ CONTEXTO } & $\begin{array}{l}\text { Relação entre o proble- } \\
\text { ma da TB e do PCT }\end{array}$ & $\begin{array}{l}\text { Problema de estrutura (física, de } \\
\text { recursos humanos - RH, desestru- } \\
\text { turação da } A B \text {, mudança no perfil } \\
\text { do doente) }\end{array}$ & $\begin{array}{l}\text { Problema social (pobreza, falta } \\
\text { meração) }\end{array}$ & de informação, drogas, aglo- \\
\hline & Influência política & \multicolumn{2}{|c|}{ Problema de gestão (alta interferência política) } & $\begin{array}{l}\text { Não se deixa influenciar } \\
\text { pela política ou não cita } \\
\text { como um problema }\end{array}$ \\
\hline & $\begin{array}{l}\text { Parceria com outros } \\
\text { setores }\end{array}$ & \multicolumn{2}{|c|}{$\begin{array}{l}\text { Dificuldade de integração com outros setores, embora existam } \\
\text { algumas parcerias na saúde }\end{array}$} & $\begin{array}{l}\text { Relatam parcerias entre } \\
\text { programas de saúde }\end{array}$ \\
\hline & $\begin{array}{l}\text { Estudos sobre as con- } \\
\text { dições de vida da po- } \\
\text { pulação }\end{array}$ & \multicolumn{3}{|c|}{$\begin{array}{l}\text { Não conhecem estudos nessa temática, mas alguns informam sua percepção do município com } \\
\text { uma baixa infraestrutura urbana e de saneamento básico }\end{array}$} \\
\hline & Impactos do Comperj & \multicolumn{2}{|c|}{$\begin{array}{l}\text { População flutuante, rede despreparada, especulação imobiliária e } \\
\text { bolsões de pobreza - população descoberta de serviços de saúde }\end{array}$} & $\begin{array}{l}\text { Não perceberam os impac- } \\
\text { tos do empreendimento } \\
\text { em suas atividades }\end{array}$ \\
\hline & Grupos vulneráveis & \multicolumn{2}{|c|}{ População carente, do tráfico, usuários de álcool e drogas } & \\
\hline \multirow{4}{*}{$\begin{array}{l}\text { PROGRAMA DE } \\
\text { CONTROLE DA } \\
\text { TUBERCULOSE }\end{array}$} & Atividades de VE da TB & $\begin{array}{l}\text { Vigilância realizada pelo PCT. O } \\
\text { setor de VE arquiva os bancos de } \\
\text { dados. Gestão fragmentada. }\end{array}$ & $\begin{array}{l}\text { VE com os bancos de dados e } \\
\text { gestão fragmentada + Traba- } \\
\text { Iho de educação em saúde e } \\
\text { para reduzir abandono }\end{array}$ & $\begin{array}{l}\text { Busca de sintomático } \\
\text { respiratório }\end{array}$ \\
\hline & $\begin{array}{l}\text { Condições para a reali- } \\
\text { zação das atividades }\end{array}$ & $\begin{array}{l}\text { Problema com os profissionais de- } \\
\text { vido à falta de autonomia da } A B \text { ou } \\
\text { mudança de perfil do profissional }\end{array}$ & \multicolumn{2}{|c|}{$\begin{array}{l}\text { Insuficientes - falta RH, recursos, baixa captação de doentes } \\
\text { e não realização das tarefas }\end{array}$} \\
\hline & & \multicolumn{3}{|c|}{$\begin{array}{l}\text { Trabalho e vínculo dos profissionais da AB Comprometimento profissional insuficiente e alta } \\
\text { rotatividade + Falta de capacitação e acolhimento }\end{array}$} \\
\hline & Recursos & $\begin{array}{l}\text { Recursos suficientes gastos por } \\
\text { demanda }\end{array}$ & \multicolumn{2}{|c|}{ Recursos insuficientes - falta carro, $\mathrm{RH}$, material e estrutura } \\
\hline
\end{tabular}




\section{Contexto}

Tal como podemos observar no quadro 2, em relação ao contexto, a TB foi associada a duas principais questões. Primeiramente, pelos profissionais da referência secundária e da $\mathrm{AB}$, como um problema relacionado a questões sociais, tais como: precárias condições de habitação, aglomeração, baixa formação escolar, empobrecimento e utilização de álcool e drogas. Para essa associação, pode-se mencionar que diversos estudos na literatura detectaram achados semelhantes, como o de Siqueira (2014). Em segundo lugar, pelos profissionais da gestão, como um problema não controlado em razão da desestruturação da $\mathrm{AB}$ do município, da falta de estrutura física e de recursos humanos capacitados para a atenção aos doentes. Dessa forma, é possível compreender que os profissionais de uma mesma categoria tendem a apresentar olhar similar sobre algumas questões, refletindo a proximidade do profissional com seu objeto de trabalho.

A totalidade dos entrevistados alegou que a instabilidade política do município foi um dos fatores que contribuíram para a desestruturação mencionada. Indicaram que as mudanças de mandatos políticos provocaram a modificação do quadro de profissionais do setor saúde. Outros estudos encontraram achados semelhantes, como o de Monroe et al. (2008), que compreende que a rotatividade dos profissionais, relacionada à descontinuidade político-partidária, prejudica a manutenção da qualificação e o estabelecimento de vínculos entre profissionais e pacientes.

A rotatividade, por sua vez, também teria contribuído para a desestruturação do setor e dificultado a realização das atividades em TB. Na fala dos entrevistados, tal fato estaria relacionado ao baixo comprometimento profissional. Considerando que permanecerão no serviço por tempo restrito, os profissionais se sentem desmotivados, com dificuldades para se comprometer ou empenhar nas atividades de trabalho, como exemplificado na fala a seguir:
Às vezes acontece, como eu já vi, boas enfermeiras sendo retiradas, realizando um bom trabalho mas retiradas porque $o$ vereador ' $x$ ' quer o enfermeiro dele, que vai fazer campanha pra ele, e coloca alguém sem compromisso nenhum porque sabe que vai ficar ali durante um tempo e depois acabou, isso é muito da política municipal.

Atrelado a isso, nota-se o entendimento parcial de alguns profissionais a respeito de suas práticas em serviço. Como exemplo, pode-se mencionar o entendimento da busca ativa de sintomáticos respiratórios (SR) como uma atividade restrita à procura de faltosos às consultas na unidade de Programa Saúde da Família (PSF) ou na referência secundária. De acordo com o manual de recomendações do Ministério da Saúde (MS) (2011), contudo, essa é a atividade de saúde pública orientada a identificar precocemente possíveis doentes.

Outro dado importante consiste na atuação dos setores do município de forma fragmentada. Os gestores alegam dificuldade de comunicação e parceria entre os próprios segmentos da saúde e, principalmente, entre os diferentes setores públicos. A intersetorialidade em saúde, entendida como a relação entre uma ou várias partes do setor saúde e uma ou várias partes de outro setor, pretende $o$ alcance dos resultados de maneira mais eficiente do que o setor saúde poderia alcançar isoladamente (SÁ ET AL., 2011), constituindo-se um desafio para o município.

Devido à realidade instalada de setores com dificuldades de comunicação, as atividades e informações não costumam ser compartilhadas. Cada grupo, departamento ou setor atua de maneira isolada e específica, dificultando a concreta articulação dos serviços para analisar a situação de vida e saúde da população com o objetivo de intervir nos problemas (MACHADO, 2011). A fala abaixo reforça essa afirmativa:

Aqui nós temos uma gestão ainda muito fragmentada, coordenações funcionando como se fossem ilhas. Falta intersetorialidade. [...] Isso é 
um desafio porque aqui tem uma cultura, como te falei, de 'esse pedaço é meu', 'eu sempre fiz assim e deu certo'.

Da mesma forma que a intersetorialidade representa uma fragilidade em Itaboraí, a realização de estudos analíticos sobre a situação de vida e saúde da população é uma lacuna. O município não tem gerado estudos e produzido análises sobre os problemas e especificidades locais, suas prioridades, necessidades e demandas. $\mathrm{O}$ processo de gestão da saúde, que pressupõe a utilização intensa da informação e do conhecimento para o direcionamento, qualificação e análise da tomada de decisão, pode perder qualidade quando não possui essas ferramentas (ALMEIDA, 1995).

Como agravante, Itaboraí passou a sofrer com os impactos da construção do Comperj. De acordo com os entrevistados, o grande afluxo de trabalhadores da construção civil sobrecarregou o sistema de saúde da região, trazendo consigo novas cargas de doença e hábitos de vida. Queixaram-se, ainda, de que a construção do complexo provocou o encarecimento dos bens de consumo e imóveis, a falta d'água, o aumento da violência urbana e dos acidentes de trânsito. Provocou o agravamento de problemas pré-existentes, como as dificuldades de acesso aos serviços públicos e privados, a falta de saneamento básico, o transporte de má qualidade e a falta de asfaltamento.

A observação direta permitiu confirmar as informações dos entrevistados. Observouse que o município de Itaboraí, apesar dos investimentos e avanços em função do estabelecimento do Comperj nos últimos nove anos, apresenta-se como um local com pouca infraestrutura urbana. As unidades onde se encontravam os serviços de gestão em saúde, como o PCT e a Secretaria Municipal de Saúde, ficavam em áreas próximas ao centro da cidade e, portanto, mais urbanizadas. As três unidades da ESF selecionadas para estudo, em contrapartida, encontravam-se em áreas predominantemente rurais. Essas áreas possuíam comércio e serviços, embora com estradas de barro e esgoto a céu aberto. O saneamento básico é um grave problema na região, e as ruas, mesmo as próximas ao centro da cidade, não são asfaltadas.

Na área da saúde, as doenças que demonstraram maior recrudescimento com a entrada do Comperj foram as do trato respiratório e as infectocontagiosas, destacando-se a dengue e a TB (TOLEDO; SABROZA, 2011). Mais especificamente em relação à TB, ocorreu a modificação do perfil de doentes que, de acordo com os entrevistados, passaram a ser, em sua maioria, usuários de drogas e álcool provenientes das áreas mais violentas da cidade. Esse perfil de doentes representa um desafio para o PCT local no que tange às atividades de vigilância e tratamento da doença.

\section{Programa de Controle da Tuberculose}

Em relação às atividades realizadas pelo PCT, destacam-se a notificação dos casos de TB, a busca de SR e a busca de contatos como as principais ações de vigilância do PCT para a contenção da doença em Itaboraí. Parte dos entrevistados relatam dificuldades na realização adequada dessas atividades por parte dos profissionais responsáveis. Tais profissionais parecem não compreender integralmente seu papel no processo de vigilância da doença, seja por desconhecer como funciona seja por questões políticas. Outros estudos na literatura, como o de Freitas et al. (2007), relatam os mesmos achados embora os relacionem à sobrecarga dos profissionais como fator promotor da falta de comprometimento ou motivação.

A busca de contatos, apesar de ser orientada pelo Programa Nacional de Controle da Tuberculose (PNCT/MS) como uma responsabilidade da ESF em Itaboraí, está sob a responsabilidade do PCT central. No município, essa atividade é realizada pela unidade de referência secundária, uma vez que as práticas de gerência e de atenção 
secundária ocorrem em consonância, em um mesmo espaço físico e com a mesma equipe de profissionais. Por meio da análise documental do livro de sintomáticos respiratórios, o 'Livro verde', notou-se que a maioria dos contatos identificados não são examinados para verificar a infecção por TB. Nas três unidades de saúde cujos dados foram estudados, 13 casos de TB foram captados. Destes 13 casos, apenas 16 dos 46 contatos identificados foram examinados.

A busca de contatos foi mencionada como uma dificuldade do programa devido à carência de recursos necessários para a atividade, tais como carro, telefone, internet, dentre outros. Achados de outros estudos corroboram sobre a carência de insumos fundamentais às práticas do PCT, como o de Cunha et al. (2015), que reforça a questão em municípios do estado do Rio de Janeiro, dentre eles o de Itaboraí.

Os principais instrumentos de registro permanecem no nível central. De acordo com os entrevistados, isto se dá para que as informações sejam registradas com maior precisão e qualidade, uma vez que a sobrecarga dos profissionais da $\mathrm{AB}$ pode provocar o prejuízo do preenchimento. O registro é uma questão-chave para o monitoramento e avaliação das atividades implantadas para o controle da doença.

$\mathrm{Na}$ análise documental, não foram identificadas metas e indicadores detalhados para a TB no município, o que pode configurar uma dificuldade para o processo de planejamento e tomada de decisão, monitoramento e avaliação das atividades relacionadas à doença. Tal realidade difere do que é orientado pelo MS no 'Manual de Recomendações para o Controle da Tuberculose' (2011).

Dessa forma, a fim de responder à primeira pergunta avaliativa deste estudo, pode-se dizer que o PCT de Itaboraí realiza as atividades de vigilância recomendadas pelo MS, embora não sejam praticadas em sua plenitude. Em relação à segunda pergunta avaliativa, considerou-se, a partir das diferentes fontes de informação utilizadas, que as atividades de vigilância realizadas no município não são suficientes para alcançar o objetivo da intervenção em questão (o PCT), que é o de controlar a doença, em razão do contexto socioeconômico e epidemiológico do município.

\section{Conclusão}

Como lições aprendidas, a TB é um problema de saúde pública em Itaboraí. No entanto, o conceito de vigilância utilizado para a TB não inclui a análise das condições de vida e saúde da população, o que torna difícil identificar as causas reais sobre as quais intervir. As parcerias estratégicas, por sua vez, são insuficientes, dada a necessidade de uma ação integrada e intersetorial.

As precárias condições de vida da população estão diretamente relacionadas à promoção da doença. Os determinantes e vulnerabilidades sociais deveriam ser o foco das ações de controle da doença. Contudo, não foram identificadas atividades específicas de planejamento e avaliação em saúde para definir estratégias que conseguissem intervir na produção e reprodução da doença no local. A perspectiva de vigilância utilizada não compreende tais análises.

A seleção das parcerias estratégicas, por sua vez, é insuficiente. A parceria realizada com a $\mathrm{AB}$, apesar de apropriada, não parece funcionar adequadamente. Um só setor, isoladamente, não é capaz de solucionar um problema de saúde pública envolvendo tantas outras questões.

Dessa forma, pode-se apresentar como sugestões: (1) o desenvolvimento de estudos e análises das situações de vida e saúde da população com a finalidade de gerar informações para a tomada de decisão; (2) a realização de salas de situação em saúde, de modo a integrar os diferentes setores de governo para discutir e promover ações intersetoriais para a resolução de problemas; e (3) a plena 
descentralização das atividades de vigilância para a $\mathrm{AB}$ em saúde, que lida diariamente com as questões do contexto local.

A flexibilização e inovação das atividades de VE favoreceriam um agir mais próximo do contexto local, atendendo às necessidades dos diferentes grupos sociais segundo suas especificidades. Compreende-se que essas ações fortaleceriam o planejamento e a avaliação em saúde, de maneira a qualificar a atenção prestada à população e as atividades de controle da doença.

São grandes os esforços realizados pelo PCT de Itaboraí para garantir boa atenção aos pacientes de TB. Tais recomendações visam, contudo, a adequação do processo de trabalho ao cenário político, social e epidemiológico local. O crescimento da utilização das informações em saúde e o fortalecimento das atividades de avaliação podem ser tarefas-chave para a aproximação da intervenção (o PCT) ao seu objetivo inicial de controlar a doença.

\section{Colaboradores}

Soares, B. C. planejou o estudo, realizou a revisão bibliográfica e a coleta e análise dos dados. Cardoso, G. P. C e Figueiró, A. C. participaram do planejamento do estudo e da análise dos dados. As três autoras trabalharam juntas em todas as etapas de elaboração do artigo.

\section{Referências}

\footnotetext{
ALMEIDA, M. F. O Uso de Informações em Saúde na Gestão dos Serviços. Saúde soc., São Paulo, v. 4, n. 1-2, p. 39-42, 1995.
}

ALVES, C. K. A. et al. Avaliação em saúde: bases bonceituais e operacionais. Rio de Janeiro: MedBook, 2010

BARDIN, L. Análise de Conteúdo. São Paulo: Edições 70, 2011;

BRASIL. Ministério da Saúde. Manual de Recomendações para o Controle da Tuberculose no Brasil. Brasília, DF: Ministério da Saúde, 2011.

Ministério da Saúde. Secretaria do Estado de

Saúde do Rio de Janeiro. Sistema de Informação de Agravos de Notificação (Sinan). Disponível em: <http:// sistemas.saude.rj.gov.br/tabnet/deftohtm.exe?sinan/ tuberculosef.def>. Acesso em: 1 dez. 2015.

BROUSSELLE, A. et al. A Análise Estratégica. In: Avaliação: conceitos e métodos. Rio de Janeiro: Editora Fiocruz; 2011, p. 95-104.

CARDOSO, G. et al. Strategic analysis of Tuberculosis prevention and control actions in Brazil and Ethiopia: One size fits all? International Journal of Public Health, Zürich, dez. 2016. Disponível em: <https://www.ncbi. nlm.nih.gov/pubmed/27990571>. Acesso em: 23 fev. 2017.

CASTELLANOS, P. L. (Coord.). Sistemas nacionales de vigilância de la situatión de salud según condiciones de vida y del impacto de las acciones de salud y bienstar. Washington, DC: OPS; OMS, 1991. 
CUNHA, N. V. et al. Estrutura, organização e processos de trabalho no controle da tuberculose em municípios do estado do Rio de Janeiro, RJ, Brasil. Interface (Botucatu), Botucatu, v. 19, n. 53, p. 251-263, 2015.

FREITAS, A. C. et al. Desvelando a vivência de uma equipe multiprofissional de residentes em saúde da família com o agente comunitário de saúde. Revista APS, Juiz de Fora, v. 10, n. 2, p. 143-155, jul./dez. 2007.

GREENE, J. C.; BENJAMIN, L.; GOODYEAR, L. The Merits of Mixing Methods in Evaluation. Evaluation, London, v. 7, n. 1, p. 25-44, 2001.

MACHADO, L. A. Construindo a intersetorialidade. Santa Catarina: Secretaria de Estado da Saúde de Santa Catarina, 2011. Disponível em: <http://portalses.saude. sc.gov.br/index.php?option=com_docman\&task=cat view\&gid=482\&Itemid=82>. Acesso em: 26 mar. 2016.

MONROE, A. A. et al. Envolvimento de equipes da Atenção Básica à Saúde no Controle da Tuberculose. Rev. esc. enferm. USP, São Paulo, v. 42, n. 2, p. 262-267, jun. 2008.

OLIVEIRA, C. M.; CASANOVA, A. O. Vigilância da Saúde no espaço de práticas da Atenção Básica. Ciênc. saúde coletiva, Rio de Janeiro, v. 14, n. 3, p. 929-936, jun. 2009.

ORGANIZAÇÃO MUNDIAL DA SAÚDE (OMS). Global Tuberculosis Report. França: OMS, 2014.

Disponível em: <http://apps.who.int/iris/ bitstream/10665/137094/1/9789241564809_eng.pdf>. Acesso em: 13 nov. 2016.
POPE, C.; MAYS, N. Métodos observacionais. In: Pesquisa qualitativa na atenção à saúde. Porto Alegre: Artmed, 2009, p. 45-56.

SÁ, L. D. et al. Intersetorialidade e vínculo no controle da tuberculose na Saúde da Família. Rev. Latino-Am. Enfermagem, São Paulo, v. 19, n. 2, p. 387-395, mar./abr. 2011.

SIQUEIRA, A. S. Determinantes socioeconômicos da produção da tuberculose: um estudo no município de Itaboraí, Região Metropolitana do Rio de Janeiro, no período de 2000 a 2011. 2014. 126 f. Tese (Doutorado em Ciência na área de Saúde Pública) - Escola Nacional de Saúde Pública Sergio Arouca, Fundação Oswaldo Cruz, Rio de Janeiro, 2014.

SOARES, B. C. Análise estratégica das ações de vigilância epidemiológica do Programa de Controle da Tuberculose do município de Itaboraí/RJ. 2015. 97 f. Dissertação (Mestrado Profissional em Sáude Pública) - Escola Nacional de Saúde Pública Sergio Arouca, Fundação Oswaldo Cruz, Rio de Janeiro, 2015.

TOLEDO, L.; SABROZA, P. C. (Coord.). Síntese Anual 2010: monitoramento da evolução das causas relevantes de adoecimento e morte nos municípios de Itaboraí, Guapimirim e Cachoeiras de Macacu: situação epidemiológica em 2010. Rio de Janeiro: Fiocruz, 2011.

Recebido para publicação em abril de 2016

Versão final em outubro de 2016

Conflito de interesses: inexistente

Suporte financeiro: não houve 